\title{
Development and chemical characterization of Casparian strips in the roots of Chinese fir (Cunninghamia lanceolata)
}

\author{
Chengwei Song ${ }^{1,2} \cdot$ Weiwei Shen ${ }^{1,2} \cdot$ Liang $\mathrm{Du}^{1,2} \cdot$ Jialong Wen ${ }^{3} \cdot$ Jinxing $\operatorname{Lin}^{1,2} \cdot$ Ruili $^{\mathrm{Li}^{1,2}}$
}

Received: 20 October 2018 / Accepted: 7 February 2019 / Published online: 15 February 2019

(c) The Author(s) 2019

\begin{abstract}
Key message Lignification and suberization of Casparian strips occurred simultaneously during the development of Cunninghamia lanceolata primary roots.

Abstract The Casparian strips in the cell walls of vascular plant root endodermis and exodermis cells are vital for the exclusion of salts and pathogens, selective nutrient uptake, and other processes related to the transport and assimilates of water. Despite the importance of Casparian strips, their chemical composition and its relevance to their diffusion barrier functions remain a matter of debate, especially in woody plants. Here, we describe the cytological features revealed by fluorescent staining of developing Casparian strips in the primary root endodermis of Chinese fir (Cunninghamia lanceolata), one of the most important timber species native to China. Using apoplastic permeability tests, we revealed that the Casparian strips provide their barrier function from the secondary stage of endodermis development, after lignin and suberin are deposited onto the surface of the protoplast next to tangential cell walls. Furthermore, we enzymatically isolated Casparian strips from the primary root endodermis and analyzed their chemical composition using Fourier transform infrared spectroscopy, revealing that they consist largely of lignin and suberin. Using confocal Raman microscopy for label-free, in situ, structural and biochemical analysis, we showed that lignin and suberin accumulated simultaneously in the developing Casparian strips. Taken together, these results elucidated Casparian strip development and characterized the lignification and suberization processes in situ in the primary roots of Chinese fir. Since this species shows broad adaptability to varying environmental conditions, our findings may facilitate work to understand the barrier function of Casparian strips in relationship to environmental stresses.
\end{abstract}

Keywords Casparian strips $\cdot$ Lignin $\cdot$ Suberin $\cdot$ In situ analysis $\cdot$ Confocal Raman microscopy $\cdot$ Cunninghamia lanceolata

Communicated by Y. Sano.

Chengwei Song and Weiwei Shen contributed equally to the manuscript.

Ruili Li

liruili@bjfu.edu.cn

$1 \quad$ Beijing Advanced Innovation Center for Tree Breeding by Molecular Design, Beijing Forestry University, Beijing 100083, China

2 College of Biological Sciences and Technology, Beijing Forestry University, Beijing 100083, China

3 College of Materials Science and Technology, Beijing Forestry University, Beijing 100083, China

\section{Introduction}

Plant roots grow in direct contact with the soil to facilitate their uptake of water and necessary solutes, but must simultaneously avoid the uptake of unwanted or toxic solutes. The roots of virtually all vascular plants have developed anatomical and physiological adaptations to exclude unwanted solutes and pathogens; these adaptations include the formation of Casparian strips in the cell walls of endodermal and exodermal cells in the primary roots (Enstone et al. 2003; Lehmann et al. 2000; Peterson et al. 1993). Casparian strips were first described by Robert Caspary (1865) as a single layer that very closely approximated cells, and were first isolated by Priestley and North (1922). They were more comprehensively illustrated by Schreiber et al. (1994), and have since been described in a wide range of plants. 
Casparian strips are a uniseriate sclerenchymatous layer that acts as a physical barrier to apoplastic transport. Casparian strips are tissue-specific and involved in blocking the nonselective apoplastic bypass flow of water (Hose et al. 2001; Steudle 2000; Steudle and Peterson 1998), preventing the uptake of ions into the stele (Nagahashi et al. 1974; Perumalla et al. 1990), and facilitating the response to different environmental stress conditions (Degenhardt and Gimmler 2000; Karahara et al. 2004; Shen et al. 2015; Yang et al. 2015). The function of Casparian strips as apoplastic transport barriers in roots has been established in many different plant species.

In most species, root endodermal cell development occurs continuously and involves three stages (Peterson 1988; Ranathunge et al. 2004; Van Fleet 1961). In the primary stage of endodermis development, lipophilic and aromatic substances are deposited in the radial and transverse walls to form the Casparian strips. In the secondary stage, suberin lamellae are deposited onto the surface of the protoplast next to the anticlinal and tangential cell walls. In the tertiary stage, cellulose is deposited on the radial walls, inner tangential walls, and transverse walls, often accompanied by lignification. Various environmental factors, such as light (Karahara and Shibaoka 1994), water (Stasovski and Peterson 1993; Enstone and; Peterson 1998), and salinity (Karahara et al. 2004), as well as phytohormones (Chen et al. 2011; Nakayama et al. 2017), have been shown to influence the formation and development of the Casparian strips.

Elucidating the chemical composition of the developing Casparian strips is vital for providing a better understanding of their structure and function. It is currently believed that Casparian bands are deposited at the intermicrofibrillar spaces of the primary cell walls, which are largely comprised of suberin, an aliphatic polyester and the main component of cork (Schreiber et al. 1994; Schreiber 1996; Zeier and Schreiber 1997), whereas other works found evidence that Casparian strips largely consist of a lignin-like polymer (Karahara et al. 2004; Schreiber et al. 1999; Yokoyama and Karahara 2001). The relative abundance of lignin and suberin in the Casparian strips varies throughout endodermal cell development, and between dicotyledonous and monocotyledonous species (Zeier et al. 1999a; Zeier and Schreiber 1998). Nevertheless, the chemical composition of the Casparian strips has remained a contentious issue. Several approaches have been used to analyze the structure and chemical composition of the Casparian strips in many plant species (Schreiber et al. 1994; Zeier and Schreiber 1999; Hartmann et al. 2002); however, traditional histological and histochemical techniques could not accurately separate the depositions of lignin and suberin from the endodermal cells. The chemical nature of the Casparian strip polymers, therefore, requires further elucidation.
In the present study, we used a combination of techniques to examine the Casparian strips of the Chinese fir (Cunninghamia lanceolata (Lamb.) Hook. (Cupressaceae)), which is native to China. Chinese fir is a dominant tree species in southern China because of its adaptability to diverse environmental conditions, and is widely planted commercially for its rapid growth and the properties of its highly durable, scented wood. This species is currently grown on over 11 million hectares (Shi et al. 2010), but its many applications mean that this area is expanding in 15 provinces across China (State Forest Administration 2014).

Here, we used fluorescent staining to observe the development of the Casparian strips under laser scanning confocal microscopy. Fourier transform infrared (FTIR) spectroscopy was used to examine the chemical composition of the Casparian strips, while confocal Raman microscopy enabled the label-free, in situ structural, and nondestructive analysis of these barriers. Confocal Raman microscopy, a combination of Raman spectroscopy and confocal microscopy, has emerged as a powerful biomedical imaging modality (Das and Agrawal 2011; Dieing and Hollricher 2008). The use of this technique has provided new insights into the chemical composition of plant cell walls in the context of their structure, making it a convenient method of quantitative chemical imaging (Agarwal 2006; Ma et al. 2011).

The purpose of this study is to examine the developmental stages and barrier functions of the endodermal Casparian strips in Chinese fir primary roots, with a special emphasis on the deposition of lignin and suberin. The results of this study should further elucidate the composition and function of Casparian strips in the primary roots of this and other tree species.

\section{Materials and methods}

\section{Plant materials and growth conditions}

Newly matured seeds were collected from Chinese fir trees in Minhou County, Fujian Province, China (N26 $35^{\prime}$, E119 $24^{\prime}, 1020$ m above sea level), on December 20, 2013 and air-dried at room temperature for 10 days. The seeds were sterilized with $0.2 \% \mathrm{NaClO}$ for $15 \mathrm{~min}$, rinsed three times with sterile water, and incubated for 1 week under continuous light at $22{ }^{\circ} \mathrm{C}$ on pledgets moistened with distilled water. The germinating seedlings were transferred to a growth chamber under a 16-h light:8-h dark photoperiod, with $500-600 \mu \mathrm{mol} \mathrm{m}{ }^{-2} \mathrm{~s}^{-1}$ photosynthetically active radiation, $22{ }^{\circ} \mathrm{C}$, and $70 \%$ relative humidity during the day, and $18{ }^{\circ} \mathrm{C}$ and $90 \%$ relative humidity at night. The seedlings were watered with sterilized distilled water every 2 or 3 days, and their primary roots reached up to $11-13 \mathrm{~cm}$ in 6 weeks under the above growth conditions. 


\section{Histochemistry of Casparian strips}

Primary roots of 6-week-old Chinese fir plants, which were approximately $8-10 \mathrm{~cm}$ long, were divided into three zones: zone I $(0-1 \mathrm{~cm})$, zone II $(1-4 \mathrm{~cm})$ and zone III $(4-8 \mathrm{~cm})$, and 0.3-cm segments were cut at 0.5, 1.0, 2.0, 4.0, 6.0, and $8.0 \mathrm{~cm}$ from the root tip. The samples were fixed with $2.5 \%$ $(\mathrm{w} / \mathrm{v})$ glutaraldehyde in $0.1 \mathrm{M}$ cacodylate buffer $(\mathrm{pH} 7.2)$ for $24 \mathrm{~h}$ at $4{ }^{\circ} \mathrm{C}$. The samples were then rinsed in $0.1 \mathrm{M}$ cacodylate buffer, dehydrated in a graded ethanol and acetone series, then infiltrated and embedded in Spurr resin (Electron Microscopy Sciences). The resin blocks were polymerized for $24 \mathrm{~h}$ at $70^{\circ} \mathrm{C}$, then cut into $10-\mu \mathrm{m}$ slices using an ultramicrotome (Leica Biosystems). The Casparian bands were stained with $0.1 \%(\mathrm{w} / \mathrm{v})$ berberine hydrochloride for $1 \mathrm{~h}$ and counter-stained with $0.5 \%(\mathrm{w} / \mathrm{v})$ aniline blue for an additional hour, as reported previously (Brundrett et al. 1988; Man et al. 2018). The stained sections were viewed under a Leica TCS SP8 confocal laser microscope (Leica Microsystems) with an excitation wavelength of $488 \mathrm{~nm}$ and a detection wavelength of $520 \mathrm{~nm}$.

\section{Permeability tests}

The apoplastic fluorescent dye calcofluor was used to test the permeability of the root endodermis cells using a previously described method (Perumalla et al. 1990; Weis et al. 2014), with some modifications. Using a razor blade, the fresh primary roots were cut into $0.5-\mathrm{cm}$ segments starting $0.5,1.0,2.0,4.0,6.0$, and $8.0 \mathrm{~cm}$ from the root tip, after which the cut edges were sealed with molten paraffin. The root segments were carefully wounded by scratching the exodermis, which is otherwise impermeable to dyes. Care was taken to ensure that only exodermis tissue was affected by this wounding, with no damage to the endodermal cell layer. The root segments were incubated in $0.01 \%$ calcofluor $(\mathrm{w} / \mathrm{v})$ for $24 \mathrm{~h}$, after which they were rinsed in phosphate buffer and sectioned using a double-edged razor blade. The sections were mounted in glycerin and directly viewed under a Leica DM2500 fluorescence microscope (Leica Microsystems). For the calcofluor-treated sections, a blue fluorescence filter was chosen, which included an exciter filter BP $365 \mathrm{~nm}$, chromatic beam filter FT $395 \mathrm{~nm}$, and barrier filter LP $420 \mathrm{~nm}$.

\section{Isolation of Casparian strips from roots}

The primary roots of 6-week-old Chinese fir seedlings were divided into two zones, zone II $(1-4 \mathrm{~cm})$ and zone III $(4-8 \mathrm{~cm})$, which were dissected into $1-\mathrm{cm}$ pieces using a razor blade. The Casparian strips were enzymatically isolated and purified following the method previously described by Schreiber et al. (1994), with some modifications. Root segments were incubated for $1 \mathrm{~h}$ at $34{ }^{\circ} \mathrm{C}$ in a separating solution containing an equal volume of $30 \%$ hydrogen peroxide and acetic acid, rinsed three times with sterile water, and then incubated at $46{ }^{\circ} \mathrm{C}$ in an enzymatic solution containing $2.5 \%(\mathrm{w} / \mathrm{v})$ cellulase (R-10; Yakult, Japan) and $1.5 \%(\mathrm{w} / \mathrm{v})$ pectinase (Y-23; Yakult) dissolved in $0.01 \mathrm{M}$ citric buffer adjusted to $\mathrm{pH} 4.8$. After about $5 \mathrm{~h}$, the root segments were rinsed three times with sterile water. The endodermal and exodermal cell walls were separated using two dissecting needles under stereomicroscopy, and the Casparian strips of the endodermis cells from zones II and III were thoroughly washed and stored in sterilized distilled water at $4{ }^{\circ} \mathrm{C}$. The isolated Casparian strips were viewed under a Leica DM2500 fluorescence microscope using a blue fluorescence filter, which contained the exciter filter BP $365 \mathrm{~nm}$, chromatic beam filter FT $395 \mathrm{~nm}$, and barrier filter LP $420 \mathrm{~nm}$.

\section{FTIR spectroscopy}

The Casparian strips isolated from the primary roots of Chinese fir were analyzed using an FTIR spectrometer (Vertex 70; Bruker, Germany). The FTIR spectra were recorded with a resolution of $2 \mathrm{~cm}^{-1}$ in the spectral range $4000-500 \mathrm{~cm}^{-1}$. The samples were scanned three times, and all data were analyzed using Origin 6.0 software.

\section{Confocal Raman microscopy}

The primary roots of 6-week-old plants were cut into $0.3-\mathrm{cm}$ segments starting $0.5,1.0,2.0,4.0,6.0$, and $8.0 \mathrm{~cm}$ from the root tip, which were then infiltrated and embedded in Spurr resin (Electron Microscopy Sciences). An ultramicrotome (Leica Biosystems) was used to cut the samples into 10- $\mu \mathrm{m}$ slices, after which the Spurr resin was dissolved in a saturated solution of sodium hydroxide in ethanol. Raman spectra were acquired using a LabRam Xplora exquisite full-automatic confocal Raman microscope (Horiba Jobin Yvon) equipped with a confocal microscope (Olympus BX51) with a motorized $x$ and $y$ axis, and a high numerical aperture (NA) microscopy objective from Olympus (MPlan $100 \times$ objective, NA $=1.40$ ). The cross-sections were focused using a linear-polarized green laser (532-nm wavelength) providing $8 \mathrm{~mW}$ of power to the sample. For mapping, an integration time of $4 \mathrm{~s}$ and steps of $0.6 \mu \mathrm{m}$ were applied. Every pixel corresponded to one scan, and each location was obtained by averaging 2 -s cycles. The wave number ranged from 3200 to $600 \mathrm{~cm}^{-1}$.

Labspect 5 software was used for the image processing and spectral analyses (Ji et al. 2013). The lignin distribution was demonstrated by integrating over the $1600 \mathrm{~cm}^{-1}$ band $\left(1550-1640 \mathrm{~cm}^{-1}\right)$, which is attributed to aromatic $\mathrm{C}=\mathrm{C}$ bond vibrations (Gierlinger and Schwanninger 2006). The suberin distribution was demonstrated by integrating over the 
$2850 \mathrm{~cm}^{-1}$ band $\left(2830-2890 \mathrm{~cm}^{-1}\right)$, which is dominated by the $\mathrm{CH}_{2}$ stretching vibration (Bernards 2002).

\section{Results}

\section{Casparian strip development in the endodermis}

To characterize the different stages of Casparian strip development in the endodermis of Chinese fir, we took cross-sections at six different positions $(0.5,1.0,2.0$, $4.0,6.0$, and $8.0 \mathrm{~cm}$ from the tip) along the primary root. Berberine-aniline blue was then used to stain the Casparian strip and the resulting green fluorescence was visualized under blue light excitation (Fig. 1). The Casparian strips were observed in the radial cell walls of endodermal cells $0.5 \mathrm{~cm}$ from the root tip (Fig. 1a), where the primary stage of endodermal cell development occurred. This signal increased $1 \mathrm{~cm}$ from the root tip, indicating that the deposition and fluorescence of Casparian strips was much greater in this region (Fig. 1b). The endodermal cells showed a thick deposition of radial walls in the region approximately $1-4 \mathrm{~cm}$ from the root tip, alongside an increase in Casparian strip signal strength (Fig. 1b-d). In the region approximately $4-8 \mathrm{~cm}$ from the root apex, the Casparian strip signal on the radial cell walls of the endodermal cells further increased in strength (Fig. 1d-f).

\section{Apoplastic permeability of Casparian strips}

We used the apoplastic tracer calcofluor, which binds to cellulose, to detect the apoplastic permeability of the root endodermis. Calcofluor could diffuse into the cortex tissue of roots with an experimentally damaged exodermis, as indicated by the blue fluorescence of their calcofluor-stained cell walls. At $0.5 \mathrm{~cm}$ from the root tip, this fluorescence was observed in the exodermis, cortex tissue, and stele (Fig. 2a). At $1 \mathrm{~cm}$ from the root tip, the blue fluorescence was only observed in the exodermis and cortex tissue below the wound and was hardly detectable in the vascular tissue (Fig. 2b).

\section{Casparian strips during endodermis development}

The cytological features of the enzymatically isolated endodermal Casparian strips from zones II ( $1-4 \mathrm{~cm})$ and III $(4-8 \mathrm{~cm})$ of the primary roots were observed using fluorescence microscopy. The Casparian strips formed continuous nets, revealed by their bright green fluorescence under blue light (Fig. 3). The cytological features of the isolated Casparian strips from primary root zones II (Fig. 3a) were similar to those of zone III (Fig. 3b), with no pit fields detected in the Casparian strips in the radial or transverse cell walls.
Fig. 1 Fluorescence images of cross-sections taken at different distances from the tip of Chinese fir primary roots. a-f Cross-sections were obtained from 7 -week-old roots at 0.5 , $1.0,2.0,4.0,6.0$, and $8.0 \mathrm{~cm}$ from the root tip and stained with berberine hemisulfate and aniline blue. Cs Casparian strips, en endodermis, $P e$ pericycle. Scale bars $25 \mu \mathrm{m}$
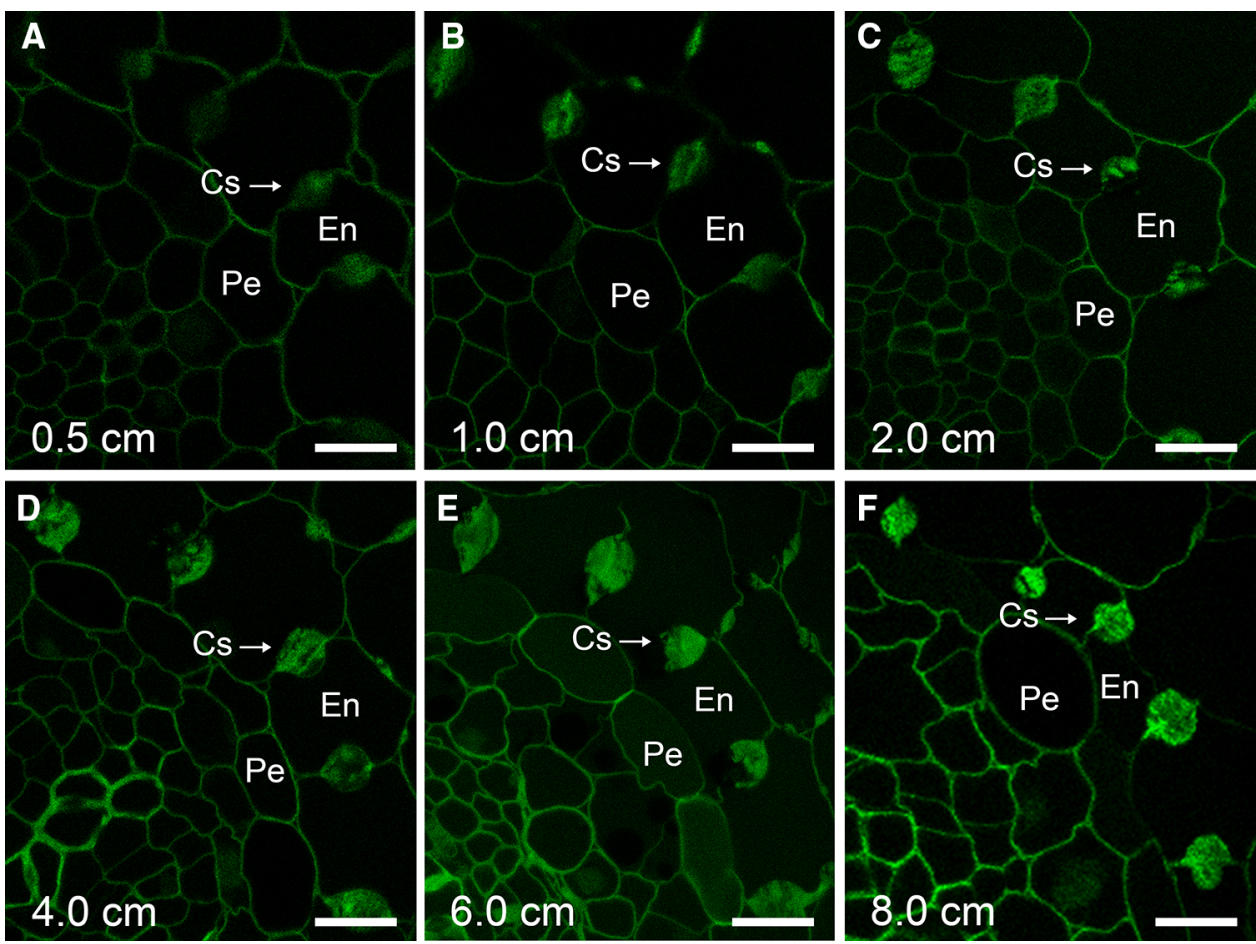
Fig. 2 Fluorescence micrographs of Chinese fir primary root cross-sections treated with calcofluor, an apoplastic tracer. a Cross-section $0.5 \mathrm{~cm}$ from the root tip treated with calcofluor. b Cross-section $1.0 \mathrm{~cm}$ from the root tip treated with calcofluor. Co cortex, En endodermis, St stele. Scale bars $100 \mu \mathrm{m}$
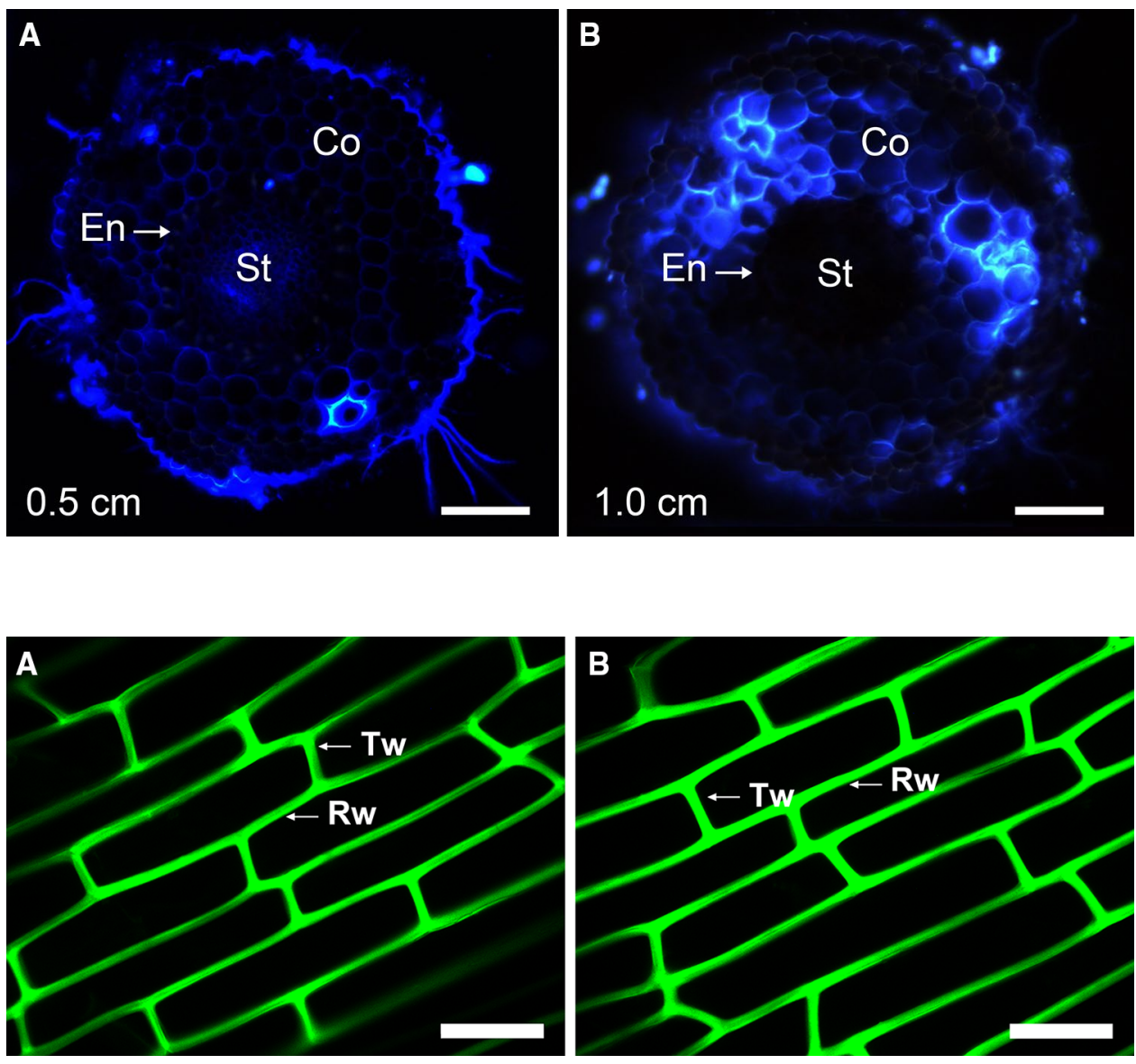

Fig. 3 Fluorescence micrographs of isolated Casparian strips from the primary roots of Chinese fir. a The isolated endodermal Casparian strips from zones II. b The isolated endodermal Casparian strips from zones III. $T w$ transverse walls of endodermal cells, $R w$ radial walls of endodermal cells. Scale bars $50 \mu \mathrm{m}$
Fig. 4 FTIR spectra of Casparian strips isolated from the primary roots of Chinese fir. The FTIR spectra were generated for Casparian strips in the secondary developmental stage (Zone II, blue) and the tertiary developmental stage (Zone III, red) of the primary root endodermis of Chinese fir

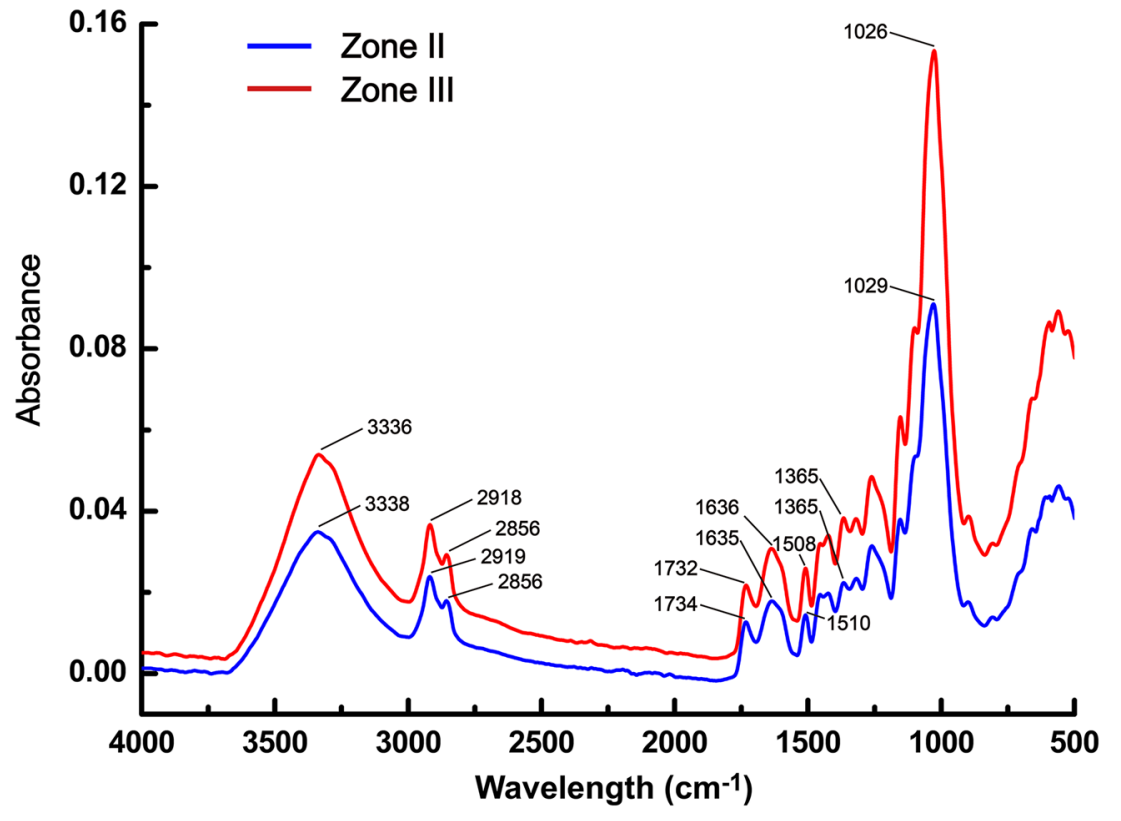




\section{Chemical composition of the Casparian strips revealed using FTIR spectroscopy}

To further identify the chemical compositions of the Casparian strips, we isolated the endodermal Casparian strip network from zones II $(1-4 \mathrm{~cm})$ and III $(4-8 \mathrm{~cm})$ of the Chinese fir primary root and scanned them with a FTIR spectrometer (Fig. 4). The spectra of the endodermal Casparian strips isolated from zones II and III of the primary roots showed similar absorption bands; however, the relative intensity of the various absorption bands were lower for zone II than for zone III, indicating that the abundances of all endodermal Casparian strip components were slightly lower in zone II.

The spectra of the Casparian strips had a broad absorption band at around $3336 \mathrm{~cm}^{-1}$, which is associated with the stretching vibrations of the hydrogen bonds in $\mathrm{OH}$ or NH groups. The two intense bands at $2918 \mathrm{~cm}^{-1}$ and $2856 \mathrm{~cm}^{-1}$ in these spectra could be assigned to the asymmetrical and symmetrical stretching vibrations of methylene groups, which are present in suberin. The strong absorption bands detected around $1732 \mathrm{~cm}^{-1}$ in the spectra from both root zones could be attributed to the stretching vibrations of the $\mathrm{C}-\mathrm{O}$ groups in suberin. The absorption bands around $1635 \mathrm{~cm}^{-1}$ and $1510 \mathrm{~cm}^{-1}$ were typical of the molecular vibrations in lignin and cellulose, respectively, while the bands around $1026 \mathrm{~cm}^{-1}$ were typical of carbohydrates such as cellulose.

\section{In situ, label-free, chemical imaging of Casparian strips using confocal Raman microscopy}

To further analyze the dynamic changes in the processes of suberification and lignification in the endodermal Casparian strips of Chinese fir, cross-sections were taken at six different positions along the primary root and imaged using confocal Raman microscopy. Two-dimensional chemical images were generated by integrating over the wavenumber ranges containing strong Raman bands. The output
Fig. 5 Confocal Raman images analysis of the endodermis from cross-sections of primary Chinese fir roots at different distances from the tip. $\mathbf{a}-\mathbf{f}$ Lignin distribution in the Casparian strips of primary roots revealed by integrating over the $1600 \mathrm{~cm}^{-1}$ band $(1550$ $\left.1640 \mathrm{~cm}^{-1}\right)$. $\mathbf{a}^{\prime}-\mathbf{f}^{\prime}$ Suberin distribution in the Casparian strips of primary roots revealed by integrating over the $2850 \mathrm{~cm}^{-1}$ band $\left(2830-2890 \mathrm{~cm}^{-1}\right)$. Crosssections were obtained at 0.5 , $1.0,2.0,4.0,6.0$, and $8.0 \mathrm{~cm}$ from the root tip. Cs Casparian strips, En endodermis. Scale bars $20 \mu \mathrm{m}$. Colour code, an increase of the signal intensity of lignin and suberin from black via blue, green, yellow to read
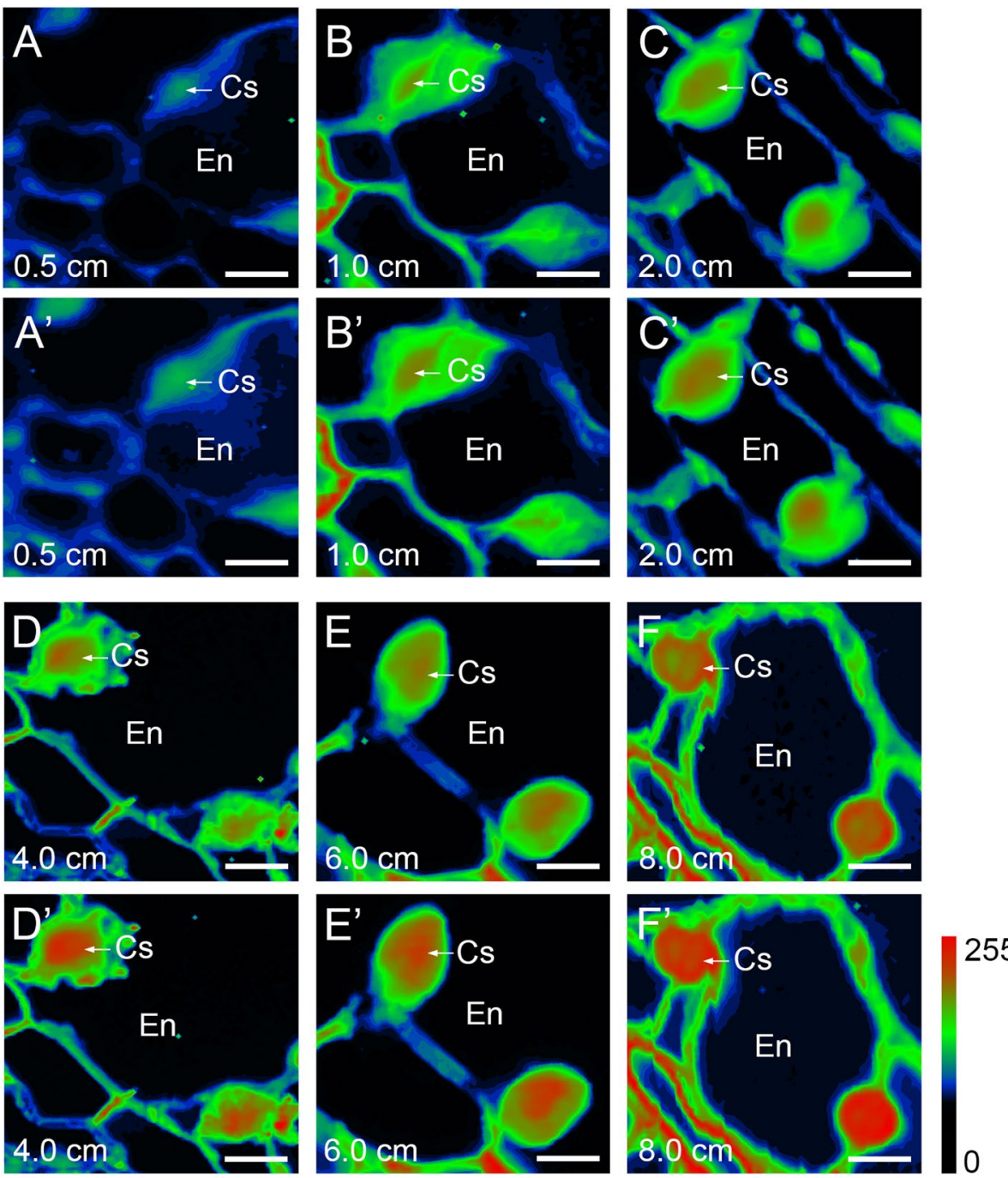
wavelengths of the optical parametric oscillators were set to about $2850 \mathrm{~cm}^{-1}$ for the suberin $\mathrm{CH}_{2}$ stretching vibrations and about $1600 \mathrm{~cm}^{-1}$ for the aromatic $\mathrm{C}=\mathrm{C}$ vibrations in lignin. When compared with the images of the tissues stained using berberine-aniline blue, the confocal Raman images of the $\mathrm{C}=\mathrm{C}$ and $\mathrm{CH}_{2}$ stretching vibrations showed a higher specificity for the lignin and suberin components of the Casparian strips, respectively (Fig. 5).

As with the berberine-aniline blue staining results, the use of confocal Raman microscopy also detected Casparian strips in the endodermal cell walls as little as $0.5 \mathrm{~cm}$ from the root tip, as illustrated by the presence of $\mathrm{C}=\mathrm{C}$ and $\mathrm{CH}_{2}$ stretching vibrations. Moreover, the confocal Raman signals of lignin and suberin became stronger in the radial cell walls of the endodermal cells $1-8 \mathrm{~cm}$ away from the root tip (Fig. 5b-f, $b^{\prime}-f^{\prime}$ ). The highest intensities of lignin and suberin were observed $8 \mathrm{~cm}$ from the root apex, indicating the maturation of the Casparian strips in the endodermis. Figure 5 shows that the confocal signal was greater in the compound middle lamella (comprising the middle lamella and adjacent primary walls) in the endodermal radial cell walls.

\section{Discussion}

The Casparian strips in the walls of root endodermis and exodermis cells are comprised of lignin and suberin in most species; however, the development of Casparian strips can be strikingly different between species (Barnabas and Peterson 1992; Enstone and Peterson 1997; Zeier et al. 1999a). In onion (Allium cepa L.) roots, Casparian strip formation begins in the center of the radial endodermal walls then expands symmetrically outwards (Barnabas and Peterson 1992), but in Arabidopsis, suberin biosynthesis and accumulation occurs much later than the formation of an endodermal barrier (Naseer et al. 2012). In this study, we used berberine-aniline blue staining and fluorescence microscopy to dissect the development of the endodermal Casparian strips in the Chinese fir primary root, revealing that they initially formed in the cell walls $0.5 \mathrm{~cm}$ from the root tip but became thicker and larger in the cells approximately $1-4 \mathrm{~cm}$ from the root tip. Endodermal cells in the region approximately 4-8 $\mathrm{cm}$ from the root tip displayed even more pronounced Casparian strips. These results suggest that the development of Casparian strips in the endodermis can be divided into three consecutive stages in the primary roots of Chinese fir.

Casparian strips form a network in the primary walls of the endodermal cells, which acts as a physical apoplastic transport barrier with important roles in the exclusion of pathogens, selective nutrient uptake, and water absorption (Julkowska and Testerink 2015; Karahara et al. 2004; Ranathunge et al. 2011). Casparian strips also prevent the radial loss of oxygen from the roots to the substrate under anoxia/ hypoxia (Kotula et al. 2009, 2014, 2017). Previously, the fluorescent dye calcofluor was used as a convenient apoplastic tracer to check the permeability of the endodermal diffusion barrier (Perumalla et al. 1990; Peterson and Perumalla 1990; Wu et al. 2005). In the present study, we demonstrated that calcofluor diffused through the endodermis and then entered the stele tissue $0.5 \mathrm{~cm}$ from the root tip, but it was hardly detectable in the root vascular tissue $1 \mathrm{~cm}$ from the tip. These results indicate that the barrier functions of the endodermis were established in the secondary development stage of the Casparian strips, when the thicker bands were observed.

The Casparian strips in the endodermal cells are impregnated with lignin and suberin, and can, therefore, be isolated by enzymatic digestion and used for microscopic and analytical investigations (Robards et al. 1976; Karahara and Shibaoka 1992). In this study, we revealed that the Casparian strips isolated from different zones of the primary roots of Chinese fir showed similar mesh morphologies. No pit fields were observed on the surface of the cell walls at the Casparian strips in the primary roots, which was also reported for Pinus bungeana (Wu et al. 2005). These results demonstrate that the continuous Casparian strip nets, form from the early stages of root development are important to provide protection from the external environment.

FTIR has been widely used to determine the chemical composition of Casparian strips, revealing that lignin and suberin are the major constituent biopolymers in many plant species (Hartmann et al. 2002; Zeier and Schreiber 1999). The conclusions made using FTIR spectroscopy have been demonstrated to be consistent with those of previous studies with direct chemical analyses performed using gas chromatography and mass spectrometry (Schreiber 1996; Schreiber et al. 1999). FTIR spectroscopy analysis of $P$. bungeana indicated that very little suberin was present in Casparian strips isolated from the needles, but equal amounts of lignin and suberin were detected in the Casparian strips of the root endodermis (Wu et al. 2005). Casparian strips isolated from the roots of three rice (Oryza sativa) cultivars were mainly composed of aliphatic suberin, lignin, and cell wall proteins and carbohydrates (Cai et al. 2011). In this study, we used FTIR spectroscopy to evaluate the chemical compositions of roots at different developmental stages, revealing that the Casparian strips in all stages had similar absorption bands at 2918, 2856, 1732, and $1636 \mathrm{~cm}^{-1}$ (Fig. 4). This indicated that the Casparian strips of the Chinese fir root endodermis at a variety of developmental stages contained aliphatic lignin and suberin, although the FTIR absorbances from Zone III were higher than Zone II at these bands (Fig. 4). This showed that the contents of lignin and suberin were higher in the Casparian strips at the tertiary stage than the secondary stage. 
The development of Casparian strips is usually accompanied with the polymerization and deposition of lignin and suberin (Chen et al. 2011; Krishnamurthy et al. 2009). Casparian strips represent only the primary developmental stage of the endodermis, which is followed by the deposition of suberin lamellae onto the surface of the protoplast next to the endodermal cell walls (Schreiber et al. 1999; Zeier et al. 1999b). Therefore, the chemical analysis of Casparian strips isolated from roots may reflect the presence of both polymers. Additionally, Schreiber et al. (1994) showed that the native structure of Casparian strips is usually destroyed by their enzymatic isolation, making their spatial analysis inaccurate. Confocal Raman microscopy overcomes this problem, as it enables their chemical composition to be assessed nondestructively and in situ, without exogenous labels, making it an appropriate technology for the investigation of Casparian strip composition at different developmental stages. We first detected the confocal Raman signals of lignin and suberin $0.5 \mathrm{~cm}$ from the root tip (Fig. 5a, a'), and their signals became stronger in the compound middle lamella of the endodermal cells $1 \mathrm{~cm}$ from the root tip, concurrent with the development and thickening of the Casparian strips (Fig. 5b, b'). The confocal Raman signal intensity and range associated with lignin and suberin were highest at $8 \mathrm{~cm}$ from the root tip. These results confirmed the findings of our berberine-aniline blue staining assay, and indicated that suberification and lignification began in the outer radial cell walls of the endodermal cells in Chinese fir, concurrent with the development of the Casparian strips. We confirmed that the endodermal cells were more permeable $0.5 \mathrm{~cm}$ from the root tip, but had formed effective barriers to water and solutes by the secondary development stage of the Casparian strips. This is consistent with previous studies obtained from Maize (Zea mays L.), which reported that the thicker deposition and larger size of Casparian dots at the secondary development stage of the Casparian strips contributed to its barrier functions (Man et al. 2018). The key functions of Casparian strips are to prevent the loss of water from the plant to their surroundings, facilitate the selective absorption of inorganic ions from the external media, and resist pathogen attack (Ma and Peterson 2003; Zeier and Schreiber 1997), making them a vital component of the root system and its ability to respond to environmental change. The early formation of the endodermal Casparian strips demonstrated here suggests that they may provide Chinese fir seedlings with a greater adaptability to the environment, which resulted in the wide distribution of this species across the various biomes in China.

In summary, we described the cytological features of the root endodermis during the development of the Casparian strips. The efficiency of their barrier function was shown to be largely related to their developmental stage and the deposition of lignin and suberin. Furthermore, using confocal
Raman microscopy, we showed that lignification and suberification occur simultaneously during Casparian strip development, which formed an effective endodermal barrier in Chinese fir.

Author contribution statement CS, WS and RL were the equal chief scientist in this study, performed the experiment, wrote the manuscript; LD participants in this study, offered some suggestions about the results, JW participants in this study, guided to complete the confocal Raman experiment; $\mathrm{RL}$ and JL designed the experiments, revised manuscripts for submission.

Acknowledgements This work was supported by the State ' 13.5 ' Key Research Program of China (no. 2016YFD0600102), the National Natural Science Foundation of China (31670182, 31761133009, 31530084, 31401149, 31501168), and the Fundamental Research Funds for the Central Universities (2017ZY10, BLX201418) and the Program of Introducing Talents of Discipline to Universities (111 project, B13007).

\section{Compliance with ethical standards}

Conflict of interest The authors declare that they have no conflict of interest.

OpenAccess This article is distributed under the terms of the Creative Commons Attribution 4.0 International License (http://creativeco mmons.org/licenses/by/4.0/), which permits unrestricted use, distribution, and reproduction in any medium, provided you give appropriate credit to the original author(s) and the source, provide a link to the Creative Commons license, and indicate if changes were made.

\section{References}

Agarwal UP (2006) Raman imaging to investigate ultrastructure and composition of plant cell walls: distribution of lignin and cellulose in black spruce wood (Picea mariana). Planta 224:1141-1153

Barnabas AD, Peterson CA (1992) Development of Casparian bands and suberin lamellae in the endodermis of onion roots. Can J Bot 70:2233-2237

Bernards MA (2002) Demystifying suberin. Can J Bot 80:227-240

Brundrett MC, Enstone DE, Peterson CA (1988) A berberine-aniline blue fluorescent staining procedure for suberin, lignin and callose in plant tissues. Protoplasma 146:133-142

Cai X, Chen T, Zhou QY, Xu L, Qu LQ, Hua XJ, Lin JX (2011) Development of Casparian strips in rice cultivars. Plant Signal Behav 6:59-65

Caspary R (1865) Remarks on the protective sheath and the formation of stem and root (translated from German). Jahrbücher für wissenschaftliche Botanik 4:101-124

Chen T, Cai X, Wu X, Karahara I, Schreiber L, Lin JX (2011) Casparian strips development and its potential function in salt tolerance. Plant Signal Behav 6:1499-1502

Das RS, Agrawal YK (2011) Raman spectroscopy: recent advancements, techniques and applications. Vib Spectrosc 57:163-176

Degenhardt B, Gimmler H (2000) Cell wall adaptations to multiple environmental stresses in maize roots. J Exp Bot 51:595-603 
Dieing T, Hollricher O (2008) High-resolution, high-speed confocal Raman imaging. Vib Spectrosc 48:22-27

Enstone DE, Peterson CA (1997) Suberin deposition and band plasmolysis in the corn (Zea mays L.) root exodermis. Can J Bot 70:1188-1199

Enstone DE, PetersonC A (1998) Effects of exposure to humid air on epidermal viability and suberin deposit ion in maize (Zea mays L.) roots. Plant Cell Environ 21:837-844

Enstone DE, Peterson CA, Ma F (2003) Root endodermis and exodermis: structure, function and responses to the environment. J Plant Growth Regul 21:335-351

Gierlinger N, Schwanninger M (2006) Chemical imaging of poplar wood cell walls by confocal Raman microscopy. Plant Physiol 140:1246-1254

Hartmann K, Peiter E, Koch K, Schubert S, Schreiber L (2002) Chemical composition and ultrastructure of broad bean (Vicia faba L.) nodule endodermis in comparison to the root endodermis. Planta 215:14-25

Hose E, Clarkson DT, Steudle E, Schreiber L, Hartung W (2001) The exodermis: a variable apoplastic barrier. J Exp Bot 52:2245-2264

Ji Z, Ma JF, Zhang ZH, Xu F, Sun RC (2013) Distribution of lignin and cellulose in compression wood tracheids of Pinus yunnanensis determined by fluorescence microscopy and confocal Raman microscopy. Ind Crop Prod 47:212-217

Julkowska MM, Testerink C (2015) Tuning plant signaling and growth to survive salt. Trends Plant Sci 20:586-594

Karahara I, Shibaoka H (1992) Isolation of Casparian strips from pea roots. Plant Cell Physiol 33:555-561

Karahara I, Shibaoka H (1994) The Casparian strips in pea epicotyls: effects of light on its development. Planta 192:269-275

Karahara I, Ikeda A, Kondo T, Uetake Y (2004) Development of the Casparian strips in primary roots of maize under salt stress. Planta 219:41-47

Kotula L, Ranathunge K, Schreiber L, Steudle E (2009) Functional and chemical comparison of apoplastic barriers to radial oxygen loss in roots of rice (Oryza sativa L.) grown in aerated or deoxygenated solution. J Exp Bot 60:2155-2167

Kotula L, Colmer TD, Nakazono M (2014) Effects of organic acids on the formation of the barrier to radial oxygen loss in roots of Hordeum marinum. Funct Plant Biol 41:187-202

Kotula L, Schreiber L, Colmer TD, Nakazono M (2017) Anatomical and biochemical characterisation of a barrier to radial $\mathrm{O}_{2}$ loss in adventitious roots of two contrasting Hordeum marinum accessions. Funct Plant Biol 44:845-857

Krishnamurthy P, Ranathunge K, Franke R, Prakash HS, Schreiber L, Mathew MK (2009) The role of root apoplastic transport barriers in salt tolerance of rice (Oryza sativa L.). Planta 230:119-134

Lehmann H, Stelzer R, Holzamer S, Kunz U, Gierth M (2000) Analytical electron microscopical investigations on the apoplastic pathways of lanthanum transport in barley roots. Planta 211:816-822

Ma F, Peterson CA (2003) Current insights into the development, structure and chemistry of the endodermis and exodermis of roots. Can J Bot 81:405-421

Ma J, hang Z, Yang G, Mao J, Xu F (2011) Ultrastructural topochemistry of cell wall polymers in Populus nigra by transmission electron microscopy and Raman imaging. BioResources 6:3944-3959

Man Y, Zhao YY, Ye R, Lin JX, Jing YP (2018) In vivo cytological and chemical analysis of Casparian strips using stimulated Raman scattering microscopy. J Plant Physiol 220:136-144

Nagahashi G, Thomson WW, Leonard RT (1974) The Casparian strips as a barrier to the movement of lanthanum in corn roots. Science 183:670-671

Nakayama T, Shinohara H, Tanaka M, Baba K, Ogawa-Ohnishi M, Matsubayashi Y (2017) A peptide hormone required for Casparian strips diffusion barrier formation in Arabidopsis roots. Science 355:284-286

Naseer S, Lee Y, Lapierre C, Franke R, Nawrath C, Geldner N (2012) Casparian strips diffusion barrier in Arabidopsis is made of a lignin polymer without suberin. Proc Natl Acad Sci USA 109:10101-10106

Perumalla CJ, Peterson CA, Enstone DE (1990) A survey of angiosperm species to detect hypodermal Casparian bands. I. Roots with a uniseriate hypodermis and epidermis. Bot J Linn Soc 103:93-112

Peterson CA (1988) Exodermal Casparian bands: their significance for ion uptake by roots. Physiol Plant 72:204-208

Peterson CA, Perumalla CJ (1990) A survey of angiosperm species to detect hypodermal Casparian bands. II. Roots with a multiseriate hypodermis or epidermis. Bot J Linn Soc 103:113-125

Peterson CA, Murrmann M, Steudle E (1993) Location of the major barriers to water and ion movement in young roots of Zea mays L. Planta 190:127-136

Priestley JH, North EE (1922) Physiological studies in plant anatomy: III. The structure of the endodermis in relation to its function. New Phytol 21:113-139

Ranathunge K, Kotula L, Steudle E, Lafitte R (2004) Water permeability and reflection coefficient of the outer part of young rice roots are differently affected by closure of water channels (aquaporins) or blockage of apoplastic pores. J Exp Bot 55:433-447

Ranathunge K, Lin J, Steudle E, Schreiber L (2011) Stagnant deoxygenated growth enhances root suberization and lignifications, but differentially affects water and $\mathrm{NaCl}$ permeabilities in rice (Oryza sativa L.) roots. Plant Cell Environ 34:1223-1240

Robards AW, Payne HL, Gunning BES (1976) Isolation of the endodermis using wall-degrading enzymes. Cytobiology 13:85-92

Schreiber L (1996) Chemical composition of Casparian strips isolated from Clivia miniata Reg. roots: evidence for lignin. Planta 199:596-601

Schreiber L, Breiner H, Riederer M, Düggelin M, Guggenheim R (1994) The Casparian strips of Clivia miniata Reg. roots: isolation, fine structure and chemical nature. Bot Acta 107:353-361

Schreiber L, Hartmann K, Skrabs M, Zeier J (1999) Apoplastic barriers in roots: chemical composition of endodermal and hypodermal cell walls. J Exp Bot 50:1267-1280

Shen J, Xu G, Zheng HQ (2015) Apoplastic barrier development and water transport in Zea mays seedling roots under salt and osmotic stresses. Protoplasma 252:173-180

Shi J, Zhen Y, Zheng R (2010) Proteome profiling of early seed development in Cunninghamia lanceolata (Lamb.) Hook. J Exp Bot 61:2367-2381

Stasovski E, Peterson CA (1993) Effects of drought and subsequent rehydration on the structure, vitality, and permeability of Allium cepa adventitious root. Can J Bot 71:700-707

State Forest Administration P. R. China (2014) Forest resources in China (2009-2013). China Forestry Publishing House, Beijing

Steudle E (2000) Water uptake by roots: effects of water deficit. J Exp Bot 51:1531-1542

Steudle E, Peterson CA (1998) How does water get through roots? J Exp Bot 49:775-788

Van Fleet DS (1961) Histochemistry and function of the endodermis. Bot Rev 27:165-221

Weis C, Hildebrandt U, Hoffmann T, Hemetsberger C, Pfeilmeier S, König C, Schwab W, Eichmann R, Hückelhoven R (2014) CYP83A1 is required for metabolic compatibility of Arabidopsis with the adapted powdery mildew fungus Erysiphe cruciferarum. New Phytol 202:1310-1319

Wu XQ, Lin JX, Lin QQ, Wang J, Schreiber L (2005) Casparian strips in needles are more solute permeable than endodermal transport barriers in roots of Pinus bungeana. Plant Cell Physiol 46:1799-1808 
Yang J, Ding C, Xu B, Chen C, Narsai R, Whelan J, Hu Z, Zhang M (2015) A Casparian strips domain-like gene, CASPL, negatively alters growth and cold tolerance. Sci Rep 5:14299

Yokoyama M, Karahara I (2001) Radial widening of the Casparian strips follows induced radial expansion of endodermal cells. Planta 213:474-477

Zeier J, Schreiber L (1997) Chemical composition of hypodermal and endodermal cell walls and xylem vessels isolated from Clivia miniata: identification of the biopolymers lignin and suberin. Plant Physiol 113:1223-1231

Zeier J, Schreiber L (1998) Comparative investigation of primary and tertiary endodermal cell walls isolated from the roots of five monocotyledoneous species: chemical composition in relation to fine structure. Planta 206:349-361

Zeier J, Schreiber L (1999) Fourier transform infrared-spectroscopic characterization of isolated endodermal cell walls from plant roots: chemical nature in relation to anatomical development. Planta 209:537-542

Zeier J, Goll A, Yokoyama M, Karahara I, Schreiber L (1999a) Structure and chemical composition of endodermal and rhizodermal/hypodermal walls of several species. Plant Cell Environ 22:271-279

Zeier J, Ruel K, Ryser U, Schreiber L (1999b) Chemical analysis and immunolocalisation of lignin and suberin in endodermal and hypodermal/rhizodermal cell walls of developing maize (Zea mays L.) primary roots. Planta 209:1-12

Publisher's Note Springer Nature remains neutral with regard to jurisdictional claims in published maps and institutional affiliations. 